\title{
Binding of microRNA-135a (miR-135a) to homeobox protein A10 (HOXA10) mRNA in a high-progesterone environment modulates the embryonic implantation factors beta3-integrin (ITGß3) and empty spiracles homeobox-2 (EMX2)
}

\author{
Xi Luo ${ }^{1,2,3,4 \#}$, Renxiang Yang ${ }^{2 \#}$, Yun $\mathrm{Bai}^{3,4}$, Lei $\mathrm{Li}^{3,4}, \mathrm{Na} \mathrm{Lin}{ }^{3,4}$, Lan Sun ${ }^{2}$, Jianjun $\mathrm{Liu}^{5}, \mathrm{Ze} \mathrm{Wu}^{3,4} \wedge$ \\ ${ }^{1}$ Faculty of Life science and Technology, Kunming University of Science and Technology, Kunming, China; ${ }^{2}$ Medical School, Kunming University \\ of Science and Technology, Kunming, China; ${ }^{3}$ Department of Reproductive Medicine, the First People's Hospital of Yunnan Province, Kunming, \\ China; ${ }^{4}$ Reproductive Medical Center of Yunnan Province, the Affiliated Hospital of Kunming University of Science and Technology, Kunming, \\ China; ${ }^{5}$ Yunnan Key Laboratory of Stem Cell and Regenerative Medicine, Research Center of Biomedical Engineering, Kunming Medical \\ University, Kunming, China \\ Contributions: (I) Conception and design: X Luo, R Yang, Z Wu; (II) Administrative support: J Liu, Z Wu; (III) Provision of study materials or \\ patients: Y Bai, L Li, N Lin; (IV) Collection and assembly of data: R Yang, L Sun; (V) Data analysis and interpretation: X Luo, J Liu, Z Wu; (VI) \\ Manuscript writing: All authors; (VII) Final approval of manuscript: All authors. \\ \#These authors contributed equally to this work. \\ Correspondence to: Ze Wu. Department of Reproductive Medicine, the First People's Hospital of Yunnan Province, Kunming, China; \\ Reproductive Medical Center of Yunnan Province, the Affiliated Hospital of Kunming University of Science and Technology, Kunming, China. \\ Email: wuzes2010@163.com; Jianjun Liu. Yunnan Key Laboratory of Stem Cell and Regenerative Medicine, Research Center of Biomedical \\ Engineering, Kunming Medical University, Kunming, China. Email: 1ju00@163.com.
}

Background: Patients with elevated circulating progesterone concentrations on the day of the human chorionic gonadotropin (hCG) trigger had relatively low implantation rates during assisted reproductive treatments. In this study, we assess the hypothesis that different concentrations of progesterone regulate the expression of homeobox protein A10 (HOXA10) and its downstream genes through miRNA-135a.

Methods: MicroRNA-135a (miR-135a), HOXA10, beta3-integrin (ITG $\beta 3)$, and empty spiracles homeobox-2 (EMX2) expression levels in endometrial tissues from patients with elevated progesterone were measured. To determine the threshold of progesterone level which can impair implantation, Ishikawa cells were used to determine the expression of the aforementioned 4 genes after exposure to 5 graded concentrations of progesterone. The dual-luciferase reporter assay was used to verify whether miR-135a regulated the expression of HOXA10. Furthermore, the effects of HOXA10 on the expression of key endometrial receptivity genes ITG $\beta 3$ and $E M X 2$ were confirmed.

Results: High progesterone levels promoted miR-135a expression in vivo, and miR-135a bound to the 3 '-untranslated region (3'-UTR) of HOXA10 mRNA to inhibit HOXA10 expression. Reduction of HOXA10 promoted EMX2 expression and inhibited ITG-3 production. Progesterone promoted the expression of HOXA10 in vitro at low concentrations. However, when the concentration was greater than $10^{-7} \mathrm{ng} / \mathrm{mL}$, progesterone inhibited HOXA10 by promoting miR-135a expression, thereby altering the expression of related genes and affecting endometrial receptivity.

Conclusions: In vitro, the trend in miR-135a expression (which first decreased and then increased) was in direct contrast to that of HOXA10 expression (which first increased and then decreased) as progesterone levels increased. The key factors regulating endometrial receptivity included ITG $\beta 3$ and EMX2, which were

\footnotetext{
^ ORCID: Xi Luo, 0000-0002-9779-3697; Ze Wu, 0000-0002-8899-0445.
} 
confirmed to be regulated by HOXA10. High progesterone levels affected miR-135a expression, and miR135a inhibited HOXA10 expression, thereby affecting endometrial receptivity.

Keywords: Empty spiracles homeobox-2 (EMX2); homeobox protein A10 (HOXA10); implantation rate; beta3integrin (ITGß3); microRNA-135a (miR-135a); progesterone elevation

Submitted Dec 30, 2020. Accepted for publication Apr 01, 2021.

doi: $10.21037 / \mathrm{atm}-21-596$

View this article at: http://dx.doi.org/10.21037/atm-21-596

\section{Introduction}

Since the advent of ART technology, more and more infertile couples have acquired their own children. In conventional ART treatment, the mature oocytes were retrieved for in vitro fertilization after control ovarian stimulation (COS), and embryos were transferred after 3-5 days of culture in vitro to obtain pregnancy. In the process of COS, it is necessary to monitor the female serum reproductive hormone levels, such as follicle stimulating hormone (FSH), estradiol (E2), luteinizing hormone (LH), progesterone, prolactin, and testosterone. Among them, the main role of progesterone is to protect the endometrium of women. During pregnancy, it can provide support and protection for the early growth and development of the fetus, and it can protect the fetus by inhibiting uterine contractions. As similar of LH in structure and function, exogenous human chorionic gonadotropin (hCG) is usually used to induce ovulation by causing artificial LH surge during ART treatment. Therefore, the main function of hCG trigger is to induce ovulation, and the serum progesterone level should be low on the day of hCG trigger. However, our previous retrospective study showed that patients with elevated progesterone levels on the day of the hCG trigger had poor outcomes with assisted reproductive technology (ART) treatment, despite non-significant differences in embryo quality among the 2,921 tested samples (1). In our more recent investigation, we further demonstrated that an elevation in plasma progesterone had differential effects on patients with different ovarian responses to hormonal stimulation (2). Hence, whole-embryo freezing and transfer of the thawed embryo are recommended for women with early elevated progesterone levels on the hCG trigger day. The intrinsic factors responsible for the ART outcome of increased progesterone on hCG trigger day has been a question we have explored.

Existing studies have shown that progesterone plays a key role in embryonic implantation (3), especially in preparing the endometrium prior to implantation and regulating the invasion of trophoblast cells and their migration into the uterine wall. The major reason for poor ART treatment outcomes is that the increase in progesterone on $\mathrm{hCG}$ trigger day in the follicular phase can negatively affect embryonic implantation (4). Even if the embryo is of high quality, the implantation process cannot be completed without optimal endometrial conditions. A primary reason may be that the high concentration of progesterone impairs endometrial receptivity (5). Progesterone modulates a series of factors affecting the endometrium, including Indian hedgehog $(I H H)(6,7)$, bone morphogenetic protein 2 (BMP2) $(8,9)$, homeobox protein A10 (HOXA10) (10), and heart and neural crest derivatives-expressed transcript 2 (HAND2) (11). These factors modulate implantation of the embryo and endometrial decidualization via progesterone receptors (PRs), which include the PR-A and PR-B subtypes. These subtypes are produced by the same DNA sequence, but are generated in response to different promoter stimuli (12).

HOXA10 is a transcription factor that contains a homeobox. In mice, HOXA10 is mainly expressed in the endometrium, and its expression is essential for mouse fertility (13). HOXA10 is also expressed in the endometrial gland and stroma throughout the human menstrual cycle, and its expression in the endometrium is related to estrogen and progesterone levels (14). During embryonic implantation, HOXA10 expression markedly increases in endometrial cells, indicating that HOXA10 plays a crucial role in the implantation of the embryo.

During the endometrial implantation window, there are two important factors in endometrial function, beta 3 -integrin (ITG $\beta 3$ ) and empty spiracles homeobox-2 $(\mathrm{Emx} 2)$ ITG $\beta 3$ is a transmembrane glycoprotein which present on the surface of cells, and is known to participate in cell adhesion as well as cell-surface-mediated signaling. Studies have shown that ITG $\beta 3$ plays an important role in 
regulating endometrial receptivity (15-17). EMX2 is a very important transcription factor in growth and development, but the high expression of EMX2 in the endometrium usually leads to abnormal development of the endometrium $(18,19)$.

MicroRNAs (miRNAs) are small non-coding endogenous RNAs that participate in gene regulation. They can control the expression of many genes, and as such, may affect multiple physiological processes. MiRNAs regulate the expression of target genes by binding to the 3 '-untranslated region (3'-UTR) of the target gene mRNA. The regulatory effect of miR-135 on HOXA10 expression in endometriosis has been previously reported by Petracco et al. (20). In our present study, we demonstrated that microRNA-135a (miR-135a) regulated HOXA10 and downstream genes involved in the endometrial implantation window in a highprogesterone environment. We present the following article in accordance with the MDAR reporting checklist (available at http://dx.doi.org/10.21037/atm-21-596).

\section{Methods}

\section{Biopsy of endometrium from patients with elevated progesterone}

This research study and protocol were approved by the Medical Ethics Committee of the First People's Hospital of Yunnan Province, China (KHLL2020-KY012). All patients signed the written informed consent form before endometrial sample collection was performed. All human research methods were conducted in accordance with relevant guidelines and regulations. All procedures performed in this study involving human participants were in accordance with the Declaration of Helsinki (as revised in 2013). Endometrium were collected from patients undergoing a conventional and standard in vitro fertilization (IVF) protocol without any additional interventions at a center licensed for ART treatments, and who had elevated progesterone on the hCG trigger day. Our sample inclusion criteria were as follows: women under 38 years of age who received IVF for the first time using a short- or long-COS protocol; patients with $<10 \mathrm{IU} / \mathrm{L}$ basal serum FSH on day 2 ; $>2.0 \mathrm{ng} / \mathrm{mL}$ serum progesterone concentration on the hCG trigger day; 8-15 oocytes retrieved; and $>80 \%$ oocyte utilization rate. 10 patients enrolled have canceled the embryo transfer and biopsied the sample of endometrium 7-8 days after oocyte retrieval using Pipelle endometrial suction curette (CooperSurgical, Trumbull, CT, USA).
The control group consisted of 10 women with normal menstruation and serum progesterone level. Endometrial biopsy was performed 7-8 days after ovulation in the natural cycle.

\section{Immunobistochemistry of endometrium in patients with elevated progesterone}

Human endometrial tissues were paraffin-embedded and sectioned onto glass slides, followed by dewaxing and rehydration of the tissue sections in xylene and graded ethanol solutions, respectively. We incubated sections with $3 \%$ hydrogen peroxide at room temperature for 10 minutes to remove endogenous peroxidases, then used citrate buffer solution to retrieve the antigens on the tissue sections. Blocking of non-specific binding sites was performed on the tissue sections using 10\% fetal bovine serum in PBS. The tissue sections were independently incubated in a humidified chamber at room temperature for 1 hour with goat antiHOXA10, mouse anti-ITG $\beta 3$, or rabbit anti-EMX2 primary antibodies, followed by incubation in a humidified chamber at room temperature for $30 \mathrm{~min}$ with rabbit anti-goat, rabbit anti-mouse, or goat anti-rabbit secondary antibodies (Abcam, Cambridge, UK), respectively. Color development of the tissue sections was performed by incubating with diluted Sav-HRP conjugates, followed by application of the 3,3'-diaminobenzidine (DAB) dye solution, and then nuclear counterstaining in hematoxylin dye for 3 minutes. We observed the distribution and expression of HOXA10, ITG $\beta 3$, and EMX2 proteins in the endometrium under a microscope (DM750, Leica, Wetzlar, Germany).

\section{Cell culture and intervention with different levels of progesterone}

We used an endometrial adenocarcinoma cell line, Ishikawa cells (21), purchased from the Procell Life Science \& Technology Co., Ltd., Wuhan, Hubei Province, China. Cells were seeded in culture wells within 24-well plates, and Dulbecco's Modified Eagle Medium (DMEM, Gibco Eggenstein, Germany) containing 10\% fetal bovine serum (FBS, Bovogen Biologicals, Essendon, VIC, Australia) and $1 \%$ streptomycin and penicillin (Thermo Fisher Scientific, Waltham, MA, USA) was used for cell culture. No additional steroid hormones were added so as to avoid affecting the cells during cell culture. However, any hormones present in the FBS might have affected our experimental results. 
In the experiment with progesterone intervention, 4 progesterone concentrations $\left(1 \times 10^{-8}, 1 \times 10^{-7}, 1 \times 10^{-6}\right.$, and $1 \times 10^{-5} \mathrm{ng} / \mathrm{mL}$ ) were added to the medium and co-cultured with the Ishikawa cells for 24 hours. This was followed by extraction of total RNA for real-time quantitative PCR (RT-qPCR) detection of miR-135a and HOXA10, ITG 33 , and EMX2 mRNA expression, and extraction of proteins for the analysis of HOXA10, ITG $\beta 3$, and EMX2 expression using western blot.

\section{Cloning the HOXA10 3'-UTR reporter gene constructs and Dual-luciferase reporter assay}

We screened all possible target genes of miR-135a using microRNA.org (http://www.microrna.org/), and our results revealed that one possible target site was the 3'-UTR of HOXA10 mRNA. Thus, to confirm whether miR-135a was bound to the mRNA region of HOXA10 to regulate gene expression, we constructed the 3'-UTR reporter of HOXA10 and its mutant using the psiCHECK ${ }^{\mathrm{TM}}-2$ vector (Promega, Fitchburg, WI, USA) (see Table S1 for primers). We then co-transfected wild-type and mutant vectors with miR-135a into the Ishikawa cell line independently, and detected firefly (hluc+) and Renilla (hRluc) luciferase activities.

\section{RT-qPCR and western blot analysis}

Total RNA of endometrium tissues and Ishikawa cells were extracted using the TRIzol reagent (Invitrogen, Carlsbad, CA, USA). The M-MLV Reverse Transcriptase First-Strand cDNA Synthesis Kit (Lucigen, Middleton, WI, USA) was used to synthesize cDNA for the detection of HOXA10 mRNA and its downstream genes ITG 33 and EMX2 (see Table S1 for primers). RT-qPCR was performed using an ABI 7500 Real-Time PCR System and the SYBR Green PCR Master Mix (Takara, Dalian, China) in a $10 \mu \mathrm{L}$ reaction system (40 reaction cycles), according to the manufacturer's instructions. The quantitative PCR procedure was as follows: $95^{\circ} \mathrm{C} 10 \mathrm{~min}$; 40 cycles of $95^{\circ} \mathrm{C} 15 \mathrm{~s}$ and $60{ }^{\circ} \mathrm{C}$ $1 \mathrm{~min}$.

Total protein was extracted from the endometrium tissues and Ishikawa cells using a pre-chilled RIPA protein lysate buffer (Thermo Fisher Scientific, Waltham, MA, USA) containing the protease inhibitor phenylmethylsulfonyl fluoride (PMSF), and protein concentration was quantified using the bicinchoninic acid (BCA) assay kit (Abcam, Cambridge, UK). A total of $30 \mu \mathrm{g}$ of sample protein was loaded onto an $8-16 \%$ polyacrylamide precast gel (SurePAGE, Jinsirui, Nanjing, China) for separation, then transferred to polyvinylidene difluoride (PVDF) membranes. Membranes were then incubated with goat anti-HOXA10 (1:1,000), rabbit anti-ITG $33(1: 1,000)$, mouse anti-EMX2 (1:1,000), and rabbit anti-GAPDH $(1: 2,000)$ primary antibodies (Abcam, Cambridge, UK). Bands were visualized using the ECL prime western blotting detection reagent (Amersham, GE Healthcare Lifesciences, Uppsala, Sweden). The Gel Doc XR+ (BioRad, Hercules, CA, USA) was used to scan the developed blots, and the protein blot results were analyzed using ImageJ software (version 1.8.0).

\section{Cell transfection and HOXA10 overexpression}

To assess whether the overexpression of HOXA10 affected the regulation of ITG $\beta 3$ and EMX2, we transfected a human HOXA10 overexpression vector with neomycin resistance (iGene Biotechnology, Guangzhou, China) into Ishikawa cells using Lipofectamine 2000 (Thermo Fisher Scientific, Waltham, MA, USA). Total RNA and proteins were extracted from the transfected cells for RT-qPCR to detect mRNA expression and for western blot to detect protein expression, respectively, of HOXA10, ITG $\beta 3$, and EMX2.

\section{Statistical analysis}

All data are presented as mean \pm standard deviation (SD). An independent $t$ test was used for comparison. One-way analysis of variance (ANOVA) followed by the Dunnett's test was used for comparisons between multiple groups. $\mathrm{P}<0.05$ was considered a statistically significant difference. All statistical analyses were performed using Prism 8 Software (GraphPad, San Diego, CA, USA).

\section{Results}

Altered expression of miR-135a, HOXA10, ITGß3, and EMX2 in the endometrium after exposure to high progesterone concentrations

RT-qPCR and western blot analysis of the endometrium demonstrated that miR-135a and EMX2 expression increased, while HOXA 10 and ITG $\beta 3$ expression decreased in endometrium epithelial cells of patients with elevated progesterone on the day of the hCG trigger. 
A
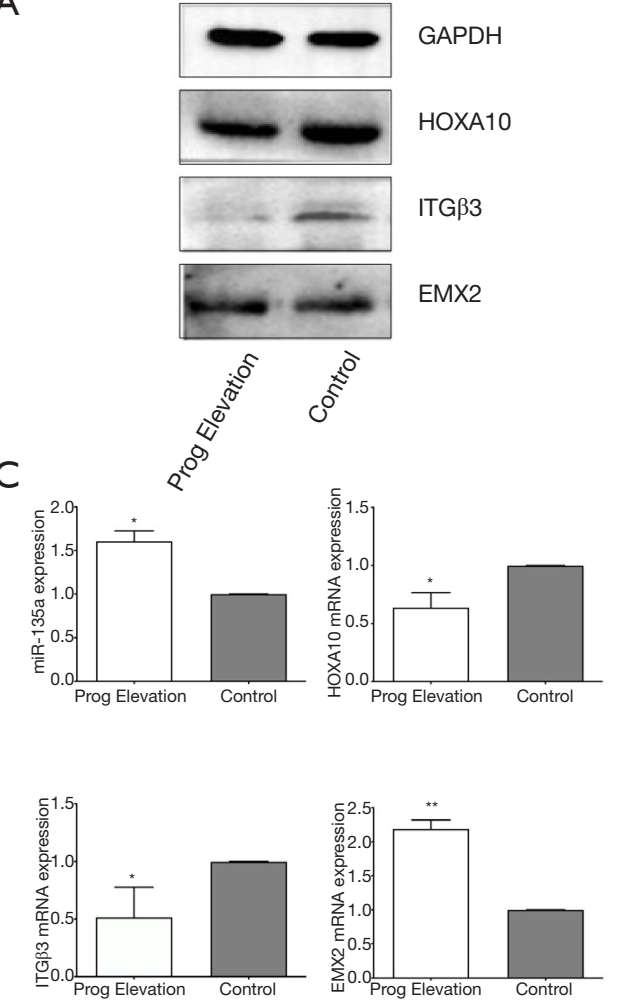

B
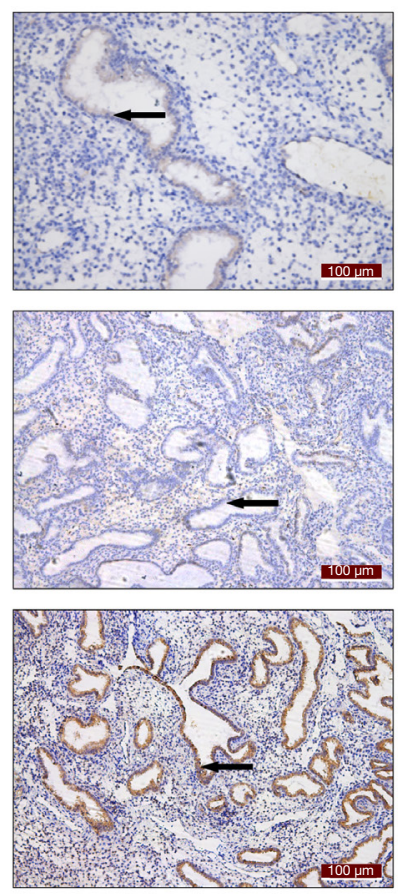

Prog Elevation
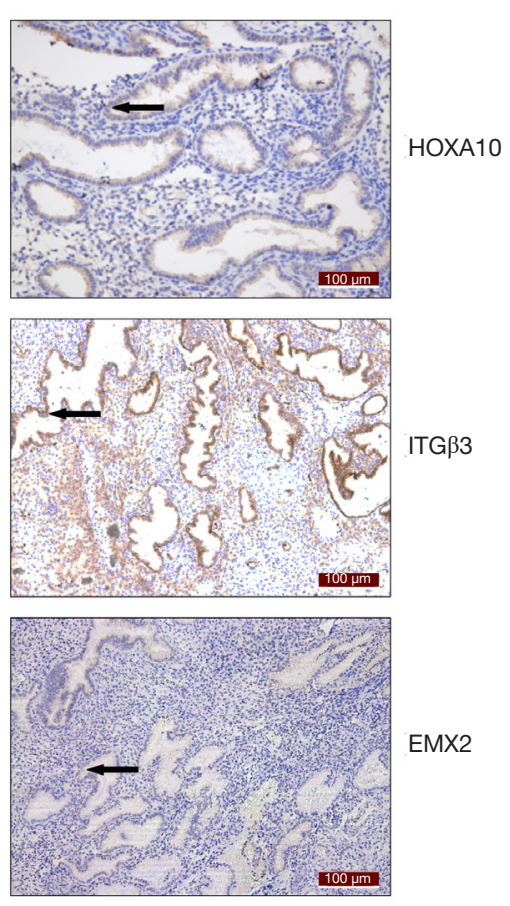

Control

EMX2

Figure 1 Analysis of endometrial tissues in patients with high progesterone levels. Representative images of (A) western blot and (B) immunohistochemical analyses of homeobox protein A10 (HOXA10), beta3-integrin (ITGB3), and empty spiracles homeobox-2 (EMX2) expression in endometrial tissues from controls and elevated progesterone (Prog Elevation) groups. (C) Real-time quantitative PCR (RT-qPCR) analysis of mRNA expression of miR-135a, HOXA10, ITG 33 , and EMX2 in endometrial tissues from control and elevated progesterone (Prog Elevation) groups (the histogram represents mean \pm standard deviation; ${ }^{*} \mathrm{P}<0.05$ and ${ }^{* *} \mathrm{P}<0.01$, with significant differences relative to the control group; $\mathrm{n}=10$ ).

Immunohistochemistry showed that HOXA10, ITG $\beta 3$, and EMX2 were primarily expressed in endometrial cells, which was consistent with the results from our western blotting analysis and RT-qPCR (Figure 1).

\section{Altered expression of miR-135a, HOXA10, ITGß3, and EMX2 in Ishikawa cells after exposure to various concentrations of progesterone}

After treating Ishikawa cells with 5 graded concentrations of progesterone, we showed that expression levels of miR135a and EMX2 were reduced after treatment with a low level of progesterone $\left(<10^{-7} \mathrm{ng} / \mathrm{mL}\right)$, while expression was enhanced in the cells treated with a high concentration of progesterone $\left(>10^{-7} \mathrm{ng} / \mathrm{mL}\right)$. HOXA10 and ITG $\beta 3$ expression levels were increased in the cells treated with a low concentration of progesterone, but were reduced in the cells treated with a high level of progesterone. These in vitro results using a high concentration of progesterone were consistent with results in the endometrium of patients with progesterone elevation. Comprehensive analysis of the expression of miR-135a, HOXA10, ITG 33 , and EMX2 after exposure to 5 graded concentrations of progesterone showed that the expression trends of miR-135a and EMX2 were in direct contrast to those of HOXA10 and ITG $\beta 3$ (Figure 2).

\section{MiR-135a regulates $\mathrm{HOX} A 10$ expression by binding to the 3'-UTR of HOXA10}

The screening results with microRNA.org showed that miR-135a regulated the expression of HOXA10 by binding to the 3'-UTR of HOXA10 mRNA, consistent with the results of the dual-luciferase reporter assay. This result was 
A

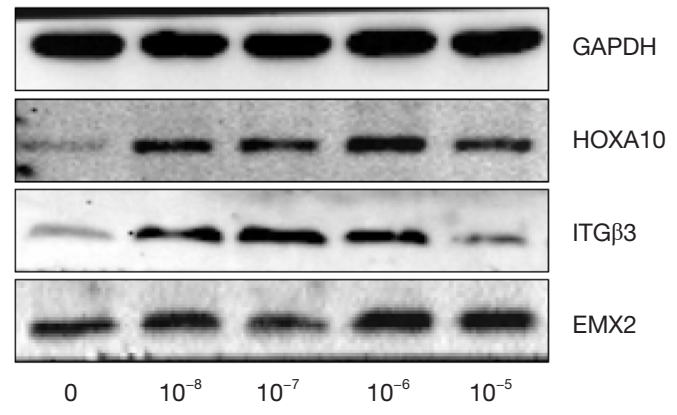

B

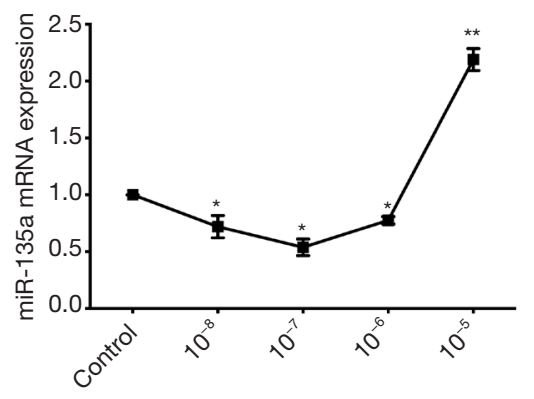

C

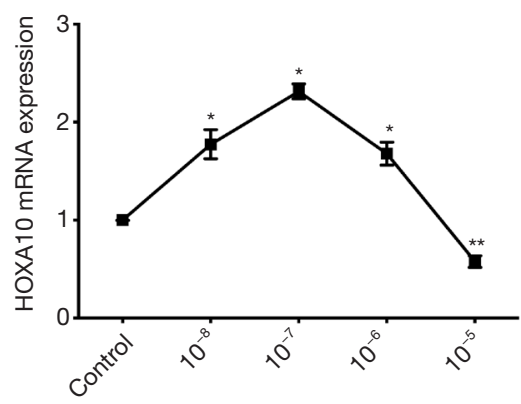

D
E

Figure 2 Analysis of microRNA-135a (miR-135a), homeobox protein A10 (HOXA10), beta3-integrin (ITG 33 ), and empty spiracles homeobox-2 (EMX2) expression in Ishikawa cells treated with various progesterone concentrations. (A) Representative images of western blot analysis of HOXA10, ITG 33 , and EMX2 expression in Ishikawa cells treated with different concentrations of progesterone for 24 hours. (B,C,D,E) Real-time quantitative PCR (RT-qPCR) analysis of mRNA expression of miR-135a (B), HOXA10 (C), ITG 33 (D), and EMX2 (E) in Ishikawa cells treated with different concentrations of progesterone for 24 hours (each dot in the charts represents the mean \pm standard deviation; ${ }^{*} \mathrm{P}<0.05,{ }^{* *} \mathrm{P}<0.01$, with significant differences relative to the control group; ns represents no significant difference; $\mathrm{n}=3$ ). Line charts represent the trend of the expression levels of different genes as the concentrations of progesterone increased. One-way analysis of variance and post-hoc analysis was used for statistical analysis.

also confirmed after mutating the 3'-UTR of HOXA10 mRNA, as miR-135a was no longer able to regulate HOXA10 expression (Figure $3 A, B$ ).

\section{MiR-135a is an inbibitor of HOXA10}

After transfecting miR-135a mimics/inhibitors into Ishikawa cells and incubating for 24 hours, HOXA10 mRNA and protein expression were measured. Our results showed that miR-135a was an inhibitory regulator of HOXA10, as an increase in miR-135a inhibited HOXA10 expression (Figure 3C,D).

\section{HOXA10 regulates the expression of ITGB3 and EMX2}

In a high-progesterone environment, low HOXA10 and ITG $\beta 3$ expression and relatively high EMX2 expression levels were observed. Our hypothesis was that HOXA10 regulated its downstream genes $I T G \beta 3$ and EMX2. After we transfected the HOXA10 overexpression vector into Ishikawa cells and incubated the cells for 24 and 48 hours, detection of mRNA and protein expression of ITG $\beta 3$ and EMX2 confirmed that HOXA10 positively regulated ITG $\beta 3$ expression and negatively regulated EMX2 expression (Figure 4).

\section{Discussion}

In the present study, we collected endometrial tissue samples from women undergoing ART treatment with abnormally elevated progesterone on the day of the hCG trigger. HOXA10, ITG $\beta 3$, and EMX2 expression varied between the tissue samples exposed to high $v s$. normal progesterone levels, and RT-qPCR also confirmed analogous mRNA expression of HOXA10, ITG 33 , and EMX2 and miR-135a levels. Our results showed that miR-135a expression was 
A

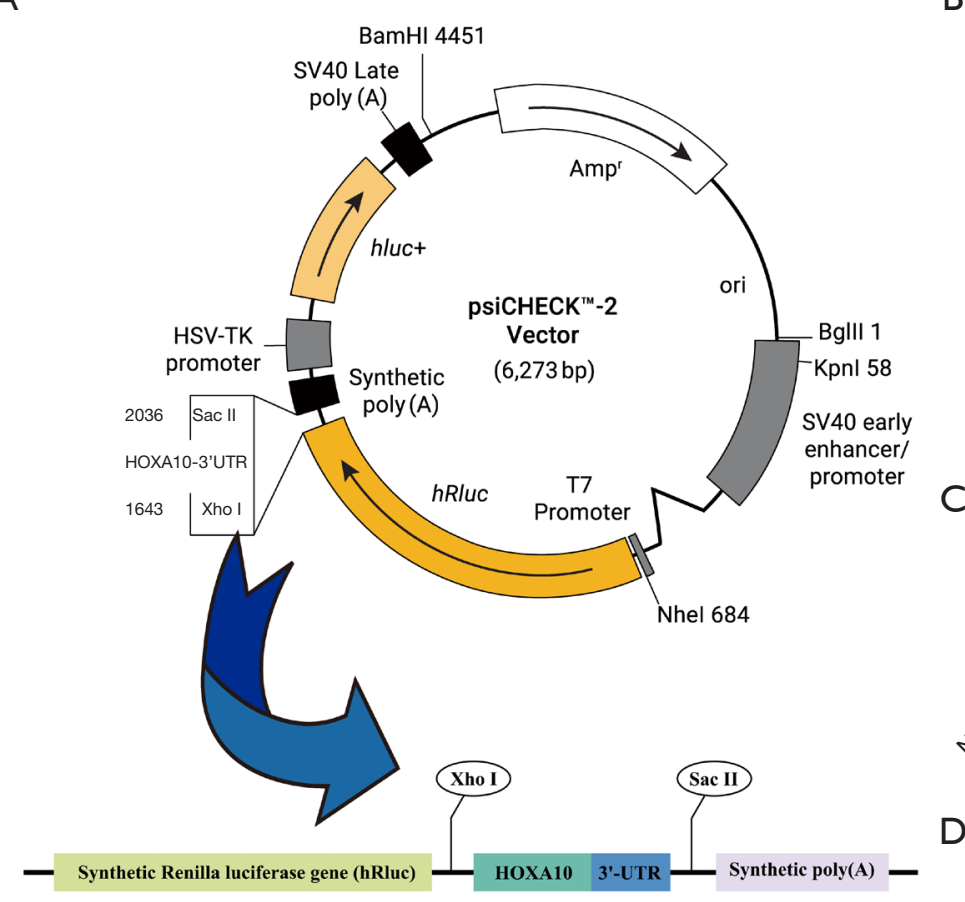

B

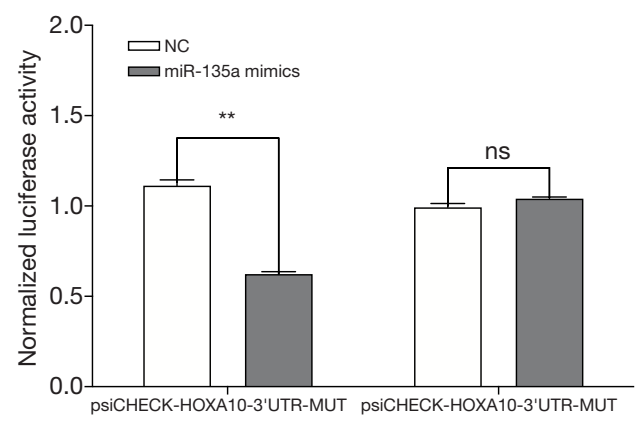

C
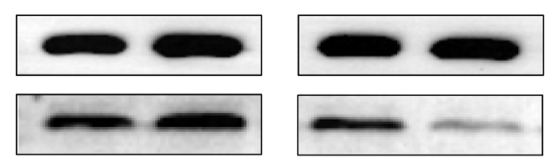

GAPDH
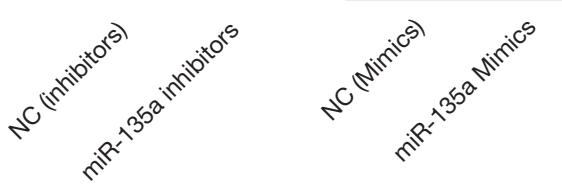

HOXA10
HOXA10 WT 207:5'-guuuucuGGGGGAAAAAGCCAUa-3'

hsa-miR-135

3'-aguguauCCUUAUUUUUCGGUAu-5'

HOXA10 MU 207:5'-guuuucuGGGGGAAACC TCCAUa-3'

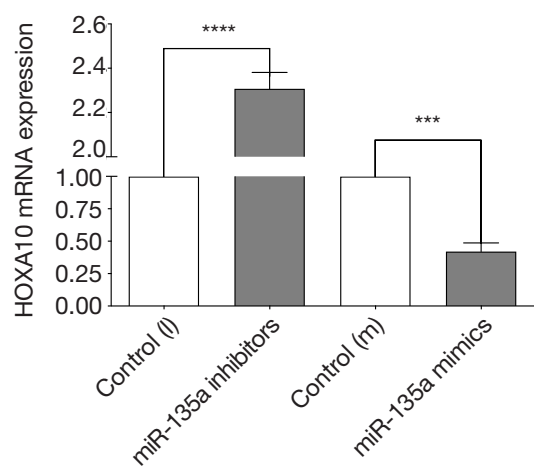

Figure 3 The dual-luciferase reporter assay demonstrated that microRNA-135a (miR-135a) regulated homeobox protein A10 (HOXA10). (A) HOXA10 mRNA 3'-UTR wild-type and mutant psiCHECK-2 vector construction. (B) Comparison of the normalized luciferase activities of Ishikawa cells transfected with psiCHECK-HOXA10-3'-UTR-WT and psiCHECK-HOXA10-3'-UTR-MUT in the negative control (NC) and the miR-135a mimic treatment groups (bar chart represents mean \pm standard deviation). (C) Representative images of western blot analysis comparing HOXA10 protein expression in Ishikawa cells transfected with miR-135a inhibitors or miR-135a mimics, and the NC. (D) Real-time quantitative PCR (RT-qPCR) analysis comparing HOXA10 mRNA expression in Ishikawa cells transfected with miR-135a inhibitors or miR-135a mimics, and the NC, to confirm the regulation of HOXA10 by miR-135a. ${ }^{* *} \mathrm{P}<0.01 ; \mathrm{n}=3$.

increased, and that HOXA10 and ITG $\beta 3$ expression levels were decreased, while EMX2 expression was increased in endometrial cells from patients with elevated progesterone. These results were also verified by immunohistochemistry. To determine the effect of progesterone concentrations on miR-135a, and on HOXA10 and its downstream gene targets $I T G \beta 3$ and $E M X 2$, we incubated Ishikawa cells with 5 graded concentrations of progesterone $\left(0,10^{-8}\right.$, $10^{-7}, 10^{-6}$, and $\left.10^{-5} \mathrm{ng} / \mathrm{mL}\right)$. After 24 hours of culture, RTqPCR and western blot analysis were used to detect the altered expression of miR-135a, HOXA10, ITG $\beta 3$, and EMX2. Our results showed that expression levels of miR135a and EMX2 were firstly suppressed and then increased, while HOXA10 and ITG 33 expression firstly increased and then declined proportional to the increase in progesterone concentration, and the cutoff was $10^{-7} \mathrm{ng} / \mathrm{mL}$. Therefore, miR-135a, HOXA10, ITGß3, and EMX2 displayed marked changes with varying concentrations of progesterone, suggesting that the level of progesterone exerts a clear regulatory effect on those genes related to the endometrium 
A

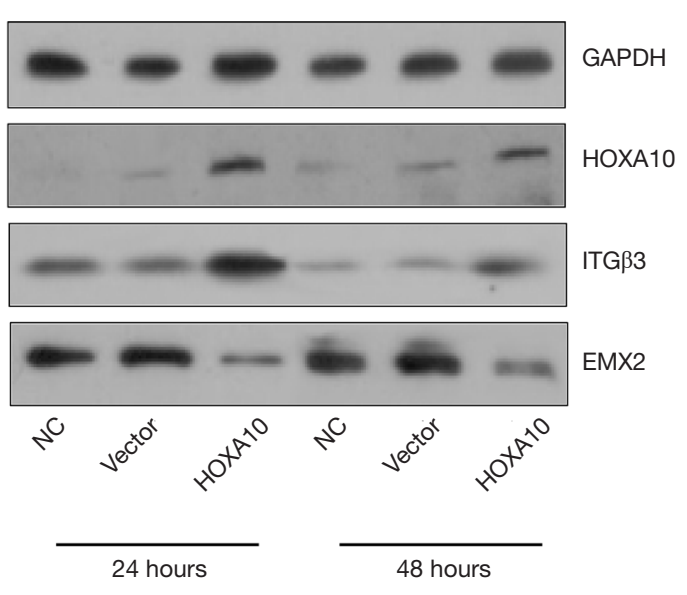

C

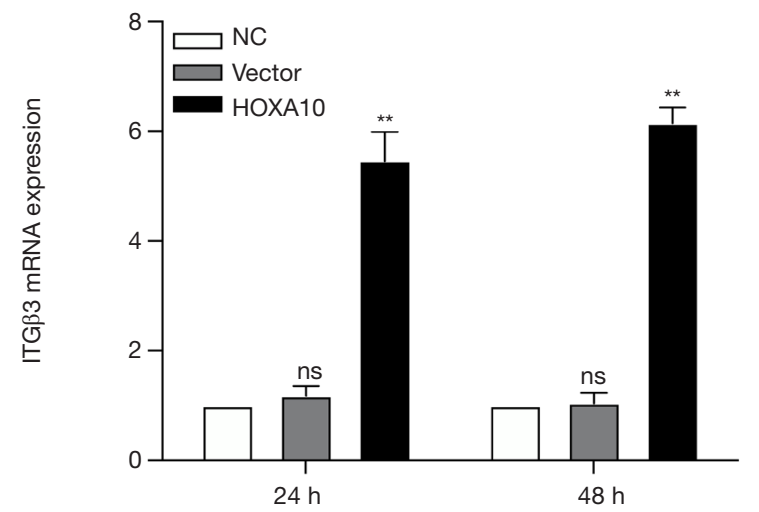

B

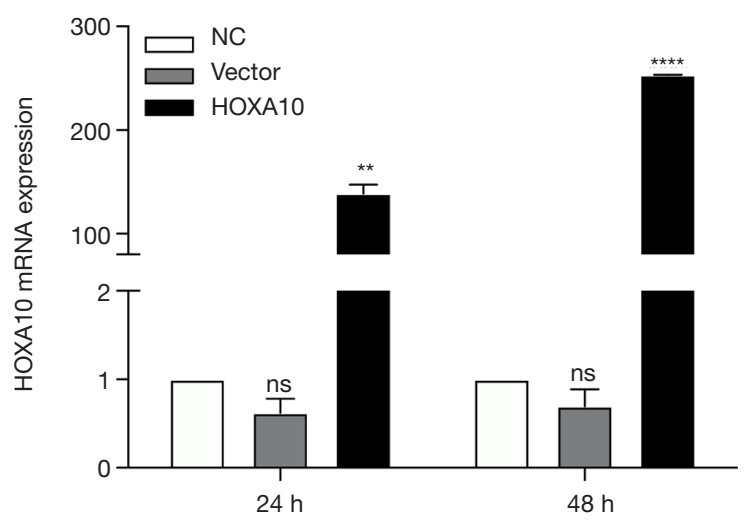

D

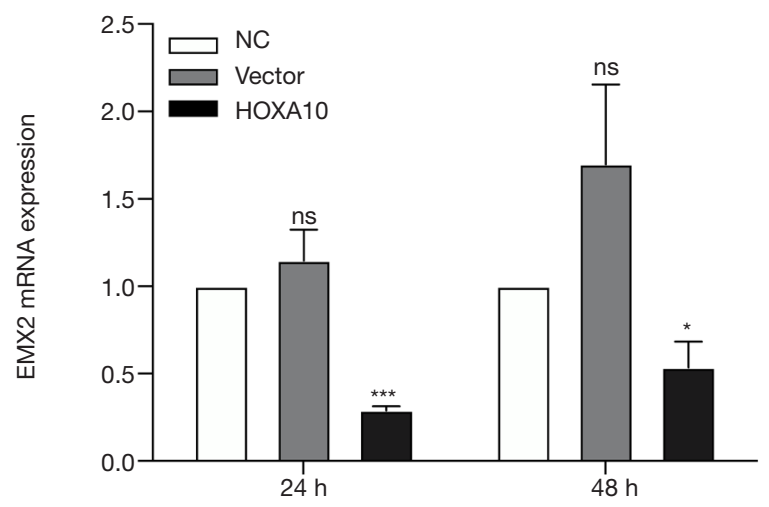

Figure 4 Validation of homeobox protein A10 (HOXA10) in the regulation of beta3-integrin (ITG $\beta 3$ ) and empty spiracles homeobox-2 (EMX2) in Ishikawa cells. (A) Representative images of western blotting of HOXA10, ITG 33 , and EMX2 expression in Ishikawa cells in the HOXA10-overexpression group, the cells of the negative control (NC) group, and the cells of the empty-vector group after 24 and 48 hours. Histograms showing (B) HOXA10, (C) ITG 33 , and (D) EMX2 mRNA expression in Ishikawa cells in the HOXA10overexpression group, the cells of the NC group, and the cells of the empty-vector group after 24 and 48 hours (the bar charts represent the mean \pm standard deviation; ${ }^{*} \mathrm{P}<0.05,{ }^{* *} \mathrm{P}<0.01$, ${ }^{* * *} \mathrm{P}<0.001$, and ${ }^{* * * *} \mathrm{P}<0.0001$, with significant differences relative to the $\mathrm{NC}$ group; ns represents no significant difference from the NS group; $\mathrm{n}=3$ ).

during the window of embryonic implantation.

HOXA10 is an important transcriptional regulator, and its expression is affected by many cytokines in different tissues and cells. In acute myeloid leukemia (AML), mixed lineage leukemia histone methylase 1 (MLL1) regulated HOXA10 expression via epigenetic changes (22). The transcription factors GATA-binding factor 1 (GATA-1) and friend leukemia integration 1 (Fli-1) also regulated the expression of human HOXA10 in megakaryocytes (23). In the study by Sarno et al. (24), preterm birth was typically caused by intraamniotic infection, with expression of proinflammatory cytokines interleukin-1 beta (IL-1 $\beta)$, or the production of decidual thrombin. HOXA10 expression was also regulated by thrombin and IL- $1 \beta$ in decidual cells from term pregnancies. Although there are numerous cytokines that exert direct or indirect effects on HOXA10, in endometrial cells, HOXA10 expression is mainly affected by progesterone. In 1998, a study on progesterone and HOXA10 by Taylor et al. (14) showed that an increase in progesterone led to elevated HOXA10 expression. In contrast, in the present study, we demonstrated that HOXA10 expression was reduced as progesterone 
concentrations increased, indicating that the relationship between progesterone and HOXA10 was nonlinear.

MiRNAs usually inhibit the continuous translation of mRNA to suppress target gene expression. In this study, we screened miRNAs through the 3'-UTR of HOXA10 mRNA, and used the dual-luciferase reporter assay for verification. Our results showed that miR-135a was strongly correlated with HOXA10, and that miR-135a expression inhibited HOXA10. Using RT-qPCR, we also demonstrated that miR-135a and HOXA10 exhibited a similar trend in expression with increased concentrations of progesterone. Similar results were also reported by Petracco et al. (20) using primary cultures of endometrial stromal cells in patients with endometriosis. Therefore, we speculated that progesterone regulated HOXA10 expression through miR135 a.

As an important transcription factor in both growth and development, EMX2 plays key roles in the development of the central nervous system (25-32), and in the development and differentiation of the reproductive system. Mice with Emx2(-/-) showed dysplasia of the reproductive system $(33,34)$. In human menstrual cycles, EMX2 transcripts are abundantly expressed in the endometrium of adults. However, compared with the proliferative phase, the mRNA levels of endometrial EMX2 decreased by $50 \%$ during embryonic implantation (18). In a study on the function of EMX2 in murine implantation, the results showed that EMX2 was negatively correlated with endometrial cell proliferation (35). These data indicate that an increase in EMX2 can interrupt the conditions for appropriate embryonic implantation, affect the intrauterine environment during the embryonic implantation window, and thereby reduce the overall rate of embryonic implantation.

Integrins are a class of transmembrane glycoproteins that are ubiquitous on the surface of cells. They are heterodimeric molecules formed by 2 non-covalent bonds of $\alpha$ and $\beta$ subunits, and specifically recognize the arginineglycine-aspartate (RGD) tripeptide sequence of ligands via their binding domains. In this way, integrins mediate cell-cell interactions, cell-extracellular matrix (ECM) interactions, and exert their 2 basic functions, adhesion and signal transduction, which are involved in physiological and pathological processes such as embryonic development, immune responses, wound repair, and metastasis of malignant tumors. Of the many factors affecting the success of embryonic implantation, endometrial receptivity is critical, as it allows the coordination of both embryonic development and endometrial status. At the beginning of embryonic implantation, trophoblast cells on the surface of the blastocyst adhere to the surface of the endometrial epithelium, and simultaneously, proteoglycans-including perlecan-appear on the blastocyst surface. Perlecan then distinguishes integrin $\alpha_{v} \beta_{3}$ and $\beta 1$ families through its core protein $(36,37)$. Therefore, ITG $\beta 3$ plays an important role in regulating overall endometrial receptivity (38-42).

In the present study, we explored the specific mechanisms underlying the reduction in embryonic implantation rates due to high progesterone based on the findings of low live birth rates in patients with elevated progesterone on the hCG trigger day. We found that a high-progesterone environment, which was more than $10^{-7} \mathrm{ng} / \mathrm{mL}$ in vitro, increased miR-135a expression, while miR-135a binding to the 3'-UTR of HOXA10 mRNA inhibited the expression of HOXA10. We also verified that HOXA10 regulated the key genes of endometrial implantation, ITG 33 and $E M X 2$. To an extent, this explains why high progesterone levels negatively affect the implantation environment and reduces the rate of embryonic implantation. However, there were still many limitations to our study. Although the results obtained by testing endometrial samples of patients with elevated progesterone were verified by in vitro experiments in Ishikawa cells, they did not fully represent the actual situation in vivo, as different individuals show varied thresholds for the effects of progesterone on embryonic implantation. Different individuals may also have different final outcomes for the same level of progesterone, and in vivo and in vitro models may manifest contrasting effects at the same progesterone level. In addition, the specific and definitive mechanisms underlying the reduction in embryonic implantation rates after progesterone elevation remain unclear. Furthermore, the specific signaling pathways that mediate the actions of PRs and miR-135a remain to be understood. Therefore, we hope to better understand the fundamental principles of human embryonic implantation by elucidating the multiple signaling pathways of PRs, combined with additional studies of the genes related to the overall process of embryonic implantation.

\section{Acknowledgments}

The authors thank all women who voluntarily provided endometrial tissue for research use.

Funding: This work was supported by the National Key Research and Development Program (Grant No. 2018YFC1002106), Yunnan Academic leaders and Reserve Personnel (Grant No. 2017HB041), Key projects 
of Basic and Applied Research in Yunnan Province (Grant No. 2018FA009), Open project of Yunnan Provincial Reproductive and Obstetrics and Gynecology Clinical Medicine Center (Grant No. 2019LCZXKF-SZ01), Program of Application and Fundamental Research of Joint Special project of Yunnan Provincial Science Technology Department \& Kunming Medical University [Grant No. 2018FE001(-302)], National Natural Science Foundation of China (Grant No. 81660266), and the Yunnan Provincial Reproductive and Obstetrics and Gynecology Clinical Medicine Center (Grant No. zx2019-01-01).

\section{Footnote}

Reporting Checklist: The authors have completed the MDAR reporting checklist. Available at http://dx.doi.org/10.21037/ atm-21-596

Data Sharing Statement: Available at http://dx.doi. org/10.21037/atm-21-596

Conflicts of Interest: All authors have completed the ICMJE uniform disclosure form (available at http://dx.doi. org/10.21037/atm-21-596). The authors have no conflicts of interest to declare.

Ethical Statement: The authors are accountable for all aspects of the work in ensuring that questions related to the accuracy or integrity of any part of the work are appropriately investigated and resolved. All experimental designs and procedures were approved by the Ethics Committee of the First People's Hospital of Yunnan Province (KHLL2020-KY012). All experimental samples were obtained with the patient's full knowledge and consent. All procedures performed in this study involving human participants were in accordance with the Declaration of Helsinki (as revised in 2013).

Open Access Statement: This is an Open Access article distributed in accordance with the Creative Commons Attribution-NonCommercial-NoDerivs 4.0 International License (CC BY-NC-ND 4.0), which permits the noncommercial replication and distribution of the article with the strict proviso that no changes or edits are made and the original work is properly cited (including links to both the formal publication through the relevant DOI and the license). See: https://creativecommons.org/licenses/by-nc-nd/4.0/.

\section{References}

1. Wu Z, Li R, Ma Y, et al. Effect of HCG-day serum progesterone and oestradiol concentrations on pregnancy outcomes in GnRH agonist cycles. Reprod Biomed Online 2012;24:511-20.

2. Wu Z, Dong Y, Ma Y, et al. Progesterone elevation on the day of hCG trigger has detrimental effect on live birth rate in low and intermediate ovarian responders, but not in high responders. Sci Rep 2019;9:5127.

3. Halasz M, Szekeres-Bartho J. The role of progesterone in implantation and trophoblast invasion. J Reprod Immunol 2013;97:43-50.

4. Kilicdag EB, Haydardedeoglu B, Cok T, et al. Premature progesterone elevation impairs implantation and live birth rates in GnRH-agonist IVF/ICSI cycles. Arch Gynecol Obstet 2010;281:747-52.

5. Venetis CA, Kolibianakis EM, Bosdou JK, et al. Progesterone elevation and probability of pregnancy after IVF: a systematic review and meta-analysis of over 60000 cycles. Hum Reprod Update 2013;19:433-57.

6. Matsumoto H, Zhao X, Das SK, et al. Indian hedgehog as a progesterone-responsive factor mediating epithelialmesenchymal interactions in the mouse uterus. Dev Biol 2002;245:280-90.

7. Takamoto N, Zhao B, Tsai SY, et al. Identification of Indian hedgehog as a progesterone-responsive gene in the murine uterus. Mol Endocrinol 2002;16:2338-48.

8. Li Q, Kannan A, Wang W, et al. Bone morphogenetic protein 2 functions via a conserved signaling pathway involving Wnt4 to regulate uterine decidualization in the mouse and the human. J Biol Chem 2007;282:31725-32.

9. Lee KY, Jeong JW, Wang J, et al. Bmp2 is critical for the murine uterine decidual response. Mol Cell Biol 2007;27:5468-78.

10. Lim H, Ma L, Ma WG, et al. Hoxa-10 regulates uterine stromal cell responsiveness to progesterone during implantation and decidualization in the mouse. Mol Endocrinol 1999;13:1005-17.

11. Li Q, Kannan A, DeMayo FJ, et al. The antiproliferative action of progesterone in uterine epithelium is mediated by Hand2. Science 2011;331:912-6.

12. Conneely OM, Lydon JP. Progesterone receptors in reproduction: functional impact of the $\mathrm{A}$ and $\mathrm{B}$ isoforms. Steroids 2000;65:571-7.

13. Satokata I, Benson G, Maas R. Sexually dimorphic sterility phenotypes in Hoxa10-deficient mice. Nature 
1995;374:460-3.

14. Taylor HS, Arici A, Olive D, et al. HOXA10 is expressed in response to sex steroids at the time of implantation in the human endometrium. J Clin Invest 1998;101:1379-84.

15. Germeyer A, Savaris RF, Jauckus J, et al. Endometrial beta3 integrin profile reflects endometrial receptivity defects in women with unexplained recurrent pregnancy loss. Reprod Biol Endocrinol 2014;12:53.

16. Liu N, Zhou C, Chen Y, et al. The involvement of osteopontin and $\beta 3$ integrin in implantation and endometrial receptivity in an early mouse pregnancy model. Eur J Obstet Gynecol Reprod Biol 2013;170:171-6.

17. Marron K, Harrity C, Dunne H, et al. Cytometric assessment of uterine receptivity via epithelial $\beta 3$ integrin expression. Reprod Biomed Online 2019;39:294-303.

18. Daftary GS, Taylor HS. EMX2 gene expression in the female reproductive tract and aberrant expression in the endometrium of patients with endometriosis. J Clin Endocrinol Metab 2004;89:2390-6.

19. Zhu Y, Luo M, Huang H, et al. HOXA10, EMX2 and TENM1 expression in the mid-secretory endometrium of infertile women with a Müllerian duct anomaly. Reprod Biomed Online 2016;32:388-93.

20. Petracco R, Grechukhina O, Popkhadze S, et al. MicroRNA 135 regulates HOXA10 expression in endometriosis. J Clin Endocrinol Metab 2011;96:E1925-33.

21. Nishida M, Kasahara K, Kaneko M, et al. Establishment of a new human endometrial adenocarcinoma cell line, Ishikawa cells, containing estrogen and progesterone receptors. Nihon Sanka Fujinka Gakkai Zasshi 1985;37:1103-11.

22. Yao J, Fang LC, Yang ZL, et al. Mixed lineage leukaemia histone methylases 1 collaborate with ERalpha to regulate HOXA10 expression in AML. Biosci Rep 2014;34:e00156.

23. Gosiengfiao Y, Horvat R, Thompson A. Transcription factors GATA-1 and Fli-1 regulate human HOXA10 expression in megakaryocytic cells. DNA Cell Biol 2007;26:577-87.

24. Sarno JL, Schatz F, Lockwood CJ, et al. Thrombin and interleukin-1beta regulate HOXA10 expression in human term decidual cells: implications for preterm labor. J Clin Endocrinol Metab 2006;91:2366-72.

25. Kimura J, Suda Y, Kurokawa D, et al. Emx2 and Pax6 function in cooperation with Otx2 and Otx1 to develop caudal forebrain primordium that includes future archipallium. J Neurosci 2005;25:5097-108.

26. Ligon KL, Echelard Y, Assimacopoulos S, et al. Loss of Emx2 function leads to ectopic expression of Wnt1 in the developing telencephalon and cortical dysplasia.

Development 2003;130:2275-87.

27. Boncinelli E, Gulisano M, Broccoli V. Emx and Otx homeobox genes in the developing mouse brain. J Neurobiol 1993;24:1356-66.

28. Yoshida M, Suda Y, Matsuo I, et al. Emx1 and Emx2 functions in development of dorsal telencephalon. Development 1997;124:101-11.

29. Pellegrini M, Mansouri A, Simeone A, et al. Dentate gyrus formation requires Emx2. Development 1996;122:3893-8.

30. Walldorf U, Gehring WJ. Empty spiracles, a gap gene containing a homeobox involved in Drosophila head development. EMBO J 1992;11:2247-59.

31. Simeone A, Acampora D, Gulisano M, et al. Nested expression domains of four homeobox genes in developing rostral brain. Nature 1992;358:687-90.

32. Simeone A, Gulisano M, Acampora D, et al. Two vertebrate homeobox genes related to the Drosophila empty spiracles gene are expressed in the embryonic cerebral cortex. EMBO J 1992;11:2541-50.

33. Pellegrini M, Pantano S, Lucchini F, et al. Emx2 developmental expression in the primordia of the reproductive and excretory systems. Anat Embryol (Berl) 1997;196:427-33.

34. Svingen T, Koopman P. Involvement of homeobox genes in mammalian sexual development. Sex Dev 2007;1:12-23.

35. Taylor HS, Fei X. Emx2 regulates mammalian reproduction by altering endometrial cell proliferation. Mol Endocrinol 2005;19:2839-46.

36. Lessey BA. Endometrial integrins and the establishment of uterine receptivity. Hum Reprod 1998;13 Suppl 3:247-58; discussion 259-61.

37. Lessey BA, Arnold JT. Paracrine signaling in the endometrium: integrins and the establishment of uterine receptivity. J Reprod Immunol 1998;39:105-16.

38. Daftary GS, Troy PJ, Bagot CN, et al. Direct regulation of beta3-integrin subunit gene expression by HOXA10 in endometrial cells. Mol Endocrinol 2002;16:571-9.

39. Yan Q, Huang C, Jiang Y, et al. Calpain7 impairs embryo implantation by downregulating beta3-integrin expression via degradation of HOXA10. Cell Death Dis 2018;9:291.

40. Zhu LH, Sun LH, Hu YL, et al. PCAF impairs endometrial receptivity and embryo implantation by down-regulating beta3-integrin expression via HOXA10 acetylation. J Clin Endocrinol Metab 2013;98:4417-28.

41. Elnaggar A, Farag AH, Gaber ME, et al. AlphaVBeta3 Integrin expression within uterine endometrium in unexplained infertility: a prospective cohort study. BMC 
Womens Health 2017;17:90.

42. He Z, Ma Y, Li L, et al. Osteopontin and integrin alphavbeta3 expression during the implantation window in IVF patients with elevated serum progesterone and oestradiol

Cite this article as: Luo X, Yang R, Bai Y, Li L, Lin N, Sun L, Liu J, Wu Z. Binding of microRNA-135a (miR135 a) to homeobox protein A10 (HOXA10) mRNA in a high-progesterone environment modulates the embryonic implantation factors beta 3 -integrin (ITG $\beta 3$ ) and empty spiracles homeobox-2 (EMX2). Ann Transl Med 2021;9(8):662. doi: $10.21037 / \mathrm{atm}-21-596$ level. Geburtshilfe Frauenheilkd 2016;76:709-17.

(English Language Editor: C. Betlazar-Maseh) 
Supplementary

Table S1 Primers used to analyze HOXA10 3'-UTR sequences using the dual-luciferase reporter assay and RT-qPCR

\begin{tabular}{|c|c|c|}
\hline Analysis & Primer & Sequence \\
\hline \multirow{3}{*}{$\begin{array}{l}\text { Primers for HOXA10 3'- } \\
\text { UTR analyzed with the } \\
\text { dual-luciferase reporter } \\
\text { assay }\end{array}$} & HOXA10 3'-UTR-wild-R & 5'-AAGCGGCCGCGCTCCTTTGCACCAT-3' \\
\hline & HOXA10 3'-UTR-mutant-F & 5'-GTTTTCTGGGGGAAAcctCCATATCATGCTAA-3' \\
\hline & HOXA10 3'-UTR-mutant-R & 5'-AGGTTTCCCCCAGAAAACAACAAATAAACCAG-3' \\
\hline \multirow{4}{*}{ RT-qPCR primers } & $\beta 3$ integrin- $R$ & 5'-GAGCAG G A CCAC CAGGAT-3' \\
\hline & EMX2-F & 5'-GGGAGAGAGAGAAAGAGAGAGAA-3' \\
\hline & EMX2-R & 5'-CCCGGTITGGTGTTGATTG-3' \\
\hline & HOXA10-F & 5'-AGGTGGACGCTGCGGCTAATCTCTA-3' \\
\hline
\end{tabular}

EMX2, empty spiracles homeobox-2; HOXA10, homeobox protein A10; RT-qPCR, real-time quantitative PCR. 